\title{
A Wireless Trigger for Synchronization of Wearable Sensors to External Systems during Recording of Human Gait
}

\author{
Patrick Kugler ${ }^{1}$, Student Member, IEEE, EMBS, Heiko Schlarb ${ }^{2}$, Joerg Blinn ${ }^{3}$, \\ Antoni Picard ${ }^{3}$, Bjoern Eskofier ${ }^{1}$, Member, IEEE, EMBS
}

\begin{abstract}
Mobile gait analysis focuses on the automatic extraction of gait parameters from wearable sensor data. However, development of algorithms for this task requires kinematic data with accurate and highly synchronous ground truth. In this paper we present a wireless trigger system which allows reliable synchronization of wearable sensors to external systems providing ground truth. To demonstrate the applicability of the system for mobile gait analysis, a Shimmer wireless sensor node with inertial sensors was mounted at the heel of a running shoe and synchronized with an external VICON motion capturing system using the wireless trigger system. Inertial sensor data were recorded during walking and running with the shoe, while kinematic and kinetic ground truth was acquired from the synchronized VICON system. Evaluation of delay and jitter of the system showed a mean delay of $2 \mathrm{~ms}$ and low jitter of 20 us. Recording was highly synchronous and the collected kinematics had a correlation of up to 0.99 . In the future the proposed system will allow the creation of a database of inertial data from human gait with accurate ground truth synchronization.
\end{abstract}

\section{INTRODUCTION}

Recently, wearable inertial sensors in the form of Body Sensor Networks (BSNs) [1] have gained high importance in human activity monitoring and gait analysis. Wireless sensor nodes like Shimmer [2] are used to detect the current activity or to provide gait parameters from inertial sensor data [3]. Such mobile motion analysis systems are also becoming important in clinical practice, e.g. for the assessment of movement disorders [4], [5]. They are also a widely used research tool in sports research or biomechanics [6], [7]. Gait parameters derived from such systems are used in many studies, e.g. for statistical comparison or to differentiate groups using classification algorithms. Examples include activities of daily living [3], the physiological state of athletes [8] or movement disorders like Parkinson's disease [9], [10].

The gold standard for motion analysis are marker based 3-D motion capturing systems, which can directly provide gait parameters like the time of foot-to-ground contact, ground clearance, stride length and step rate. However, when using mobile sensor systems for gait analysis, specialized algorithms are required to estimate the parameters from the inertial sensor data [11]. In order to develop and evaluate such algorithms, synchronous ground truth with inaccuracies in the millisecond range are needed. This is a challenging

This work was supported by the adidas AG, Herzogenaurach, Germany, the Bavarian Ministry for Economic Affairs and the European Fund for Regional Development. Corresponding author: Patrick Kugler (patrick.kugler@cs.fau.de)

${ }^{1}$ P. Kugler and B. Eskofier are with the Digital Sports Group, Pattern Recognition Lab, Computer Science Department, Friedrich-Alexander University of Erlangen-Nuremberg, Erlangen, Germany.

${ }^{2} \mathrm{H}$. Schlarb is with the adidas innovation team (a.i.t.), adidas AG, Herzogenaurach, Germany.

${ }^{3}$ J. Blinn and A. Picard are with the Department of Computer Sciences and Microsystems Technology, University of Applied Sciences Kaiserslautern, Zweibruecken, Germany. task for BSNs, as wireless transmission can introduce unpredictable delays [1].

One possible solution for this problem is to employ a clock synchronization, which is a standard task in wireless sensor networks [1], [12]. Examples are the Berkeley algorithm, Network Time Protocol and Precision Time Protocol [12]. Protocols specifically designed for wireless sensor networks are the Lightweight Time Synchronization Protocol, Reference Broadcast Synchronization, the Flooding Time Synchronization Protocol or Hierarchy Referencing Time Synchronization [12]. However, these protocols require a estimation of the wireless delay, which is usually done by exchanging messages. Hence an active bidirectional link is required at all times, which reduces battery life and reduces the communication bandwidth available for data transmission. Clock synchronization is also only provided between nodes of the network (internal time synchronization) and not with an external system (external event synchronization). Hence an additional node for the external system is required. This introduces further cost and an additional point of failure, especially as the subject must stay in communication range.

Full clock synchronization is also not required for synchronous recording of kinematic data. Instead it would be enough if the external system would send a synchronization signal to the mobile sensor node. For this a separate wireless link with known delay and very small jitter could be used to send a short trigger pulse, e.g. when the subject crosses a light barrier (event synchronization), or by sending a signal at regular time intervals (continuous synchronization). Such a system would enable the synchronous recording of kinematic of human motion while at the same time providing high accuracy ground truth from an external system.

The purpose of this paper is to present a wireless trigger system connected to a wearable sensor that allows recording of human gait with high accuracy ground truth synchronization. We thoroughly evaluate the trigger delay and compare measured kinematics for walking and running to a gold standard system. In the future the proposed system will allow the creation of a database of inertial sensor data from human gait with accurate ground truth synchronization.

\section{METHODS}

The main components of the system are a wireless trigger system and a modified Shimmer sensor node mounted to the heel of a running shoe (Fig. 1 and 2).

\section{A. Wireless Trigger System}

The wireless trigger system is based on a commercial trigger system (Yongnuo, Hong Kong, P.R. China), which is usually used to synchronously trigger multiple photographic 


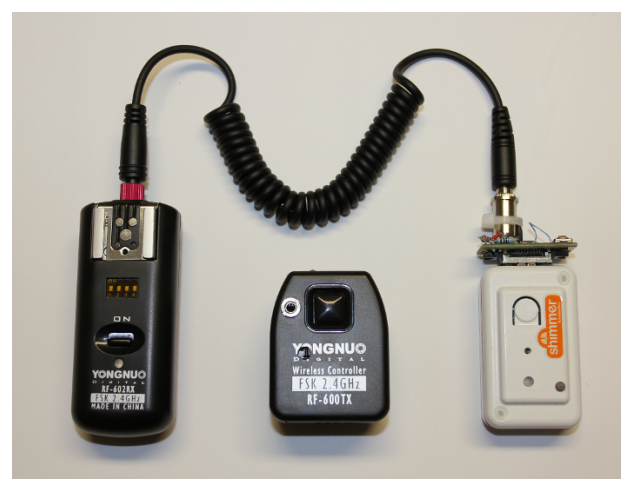

Fig. 1. The main components of the wireless system: trigger receiver (left), trigger transmitter (middle) and modified Shimmer sensor node (right).

flashlights from a single camera. The photographic application is similar to the task of external event synchronization with respect to the requirements on latency and jitter. The system consisted of a RF-600TX transmitter and one or more RF-602RX receivers (Fig. 1 left and right). The system was chosen as it was portable and reasonably small.

To adapt the system some modifications were necessary. The transmitter was fitted with a connector for the input trigger signal and modified to support a range of input voltages. A power switch was integrated and the button was modified to allow manual triggering. The receiver was also modified to be compatible with the employed sensor node. All modifications were fitted into the original housings.

The final wireless trigger system can be connected to any external system that provides a synchronization signal. When an external trigger pulse is sent to the input of the transmitter, a short coded signal is transmitted in the $2.4 \mathrm{GHz}$ band. This signal is received by all receivers, which generate a $100 \mathrm{~ms}$ trigger output signal.

\section{B. Shimmer Sensor Node}

To enable recording of kinematic data, a sensor node from the Shimmer BSN development platform [2] (Realtime Technologies Ltd., Dublin, Ireland) was employed (Fig. 1 middle). The battery powered nodes had a low power MSP430F1611 microcontroller (Texas Instruments, Dallas, Texas, USA) and Bluetooth wireless connectivity. The system was chosen as it was small and lightweight and could be easily extended with additional sensors.

The node integrated an MMA7260Q 3-axis accelerometer (Freescale Semiconductors, Austin, Texas, USA) with $6 \mathrm{~g}$ range. In contrast to standard configuration, the sensor node was additionally equipped with IDG-650 and ISZ650 gyroscopes (InvenSense, Sunnyvale, California, USA), which provided 3 -axis angular velocities with a maximum range of $2000 \%$ s. This modification was done as the default gyroscopes only provided a range of $500 \%$, which was not enough to capture the high angular velocities during running. Together this allowed recording of inertial sensor data with 6 degrees of freedom (6-DOF).

The sensor node was modified to allow connection of the wireless trigger receiver to an input port. To include the trigger signal into the data collection, the original open source firmware was modified to sample and record the trigger signal along with the other signals and to transmit all data to a PC using Bluetooth. This allowed exact association between the trigger signal and the recorded sensor data.

\section{Running Shoe Integration}

To create a wearable system for the recording of human foot kinematics, the sensor node was mounted to the heel of a running shoe (Fig. 2). An adidas adiZERO ${ }^{T M}$ Boston running shoe (adidas AG, Herzogenaurach, Germany) was modified with a special heel cap to firmly attach the node to the posterior side of the right heel. The trigger receiver was fixed to the ankle of the subject using a Velcro band and connected to the sensor node using a flexible cable.

To allow recording of kinematic ground truth with an external VICON (Oxford Metrics Group, Oxford, UK) motion capturing system, additionally three reflective markers were added to the posterior, medial and lateral sides of the sensor node to form a triangle in the transversal plane (Fig. 2).

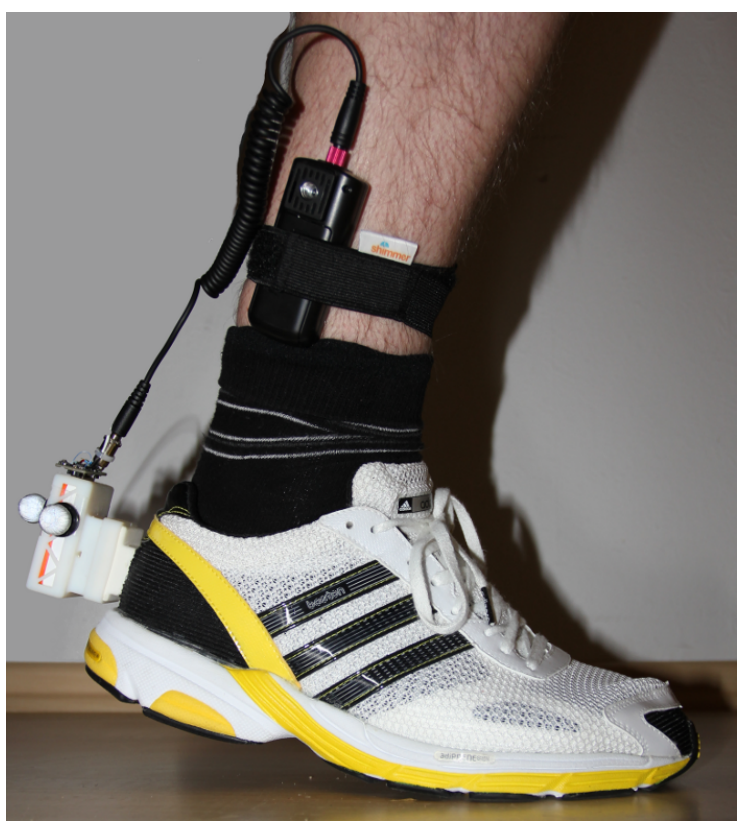

Fig. 2. Setup for synchronous kinematic recording. A Shimmer node was mounted to the heel of a running shoe and connected to a trigger receiver fixed at the ankle. Three reflective markers were added for evaluation.

\section{EVALUATION}

\section{A. Trigger Accuracy}

The latency and jitter of the wireless synchronization system were evaluated before using the system for kinematic recording. A function generator was used to generate a series of trigger pulses at a frequency of $1 \mathrm{~Hz}$. These pulses were received by three modified trigger receivers $(\mathrm{R} 1, \mathrm{R} 2$ and $\mathrm{R} 3)$ over a time period of 15 minutes, amounting to over 900 triggers. This test was done separately for a total of three transmitters (T1, T2 and T3). All signals were connected to an oscilloscope (Tektronix Inc., Beaverton, Oregon, USA), which was used to calculate the mean and standard deviation of the trigger delay for all transmitter-receiver combinations.

\section{B. Foot Kinematics}

To perform an evaluation in a realistic setting, the system was used for synchronous recording of human gait. A single subject wearing the instrumented shoe performed a total of 8 trials each while walking (at $1.8 \mathrm{~m} / \mathrm{s}$ ) and running (at 4 $\mathrm{m} / \mathrm{s}$ ) along a predefined track. Running speed was controlled 
using a light barrier. During the trials 6-DOF inertial sensor data were recorded from the Shimmer sensor. At the same time the VICON system was used to record the motion of the three reflective markers. Both systems recorded at a sampling rate of $200 \mathrm{~Hz}$. Angular velocity signals were filtered with a 10th-order Butterworth low-pass filter with $100 \mathrm{~Hz}$ cutoff frequency to reduce noise. Additional force data were recorded synchronously at $2000 \mathrm{~Hz}$ from a force plate (Kistler, Winterthur, Switzerland). A light barrier at the beginning of the capture volume was used to synchronously start the VICON recording and the wireless transmitter.

For each trial, a single step was extracted from both the VICON and Shimmer recordings. Each step started at the trigger signal and was cut to $600 \mathrm{~ms}$ for running and $800 \mathrm{~ms}$ for walking. Foot-to-ground contact time was extracted from the force plate data using a threshold of $10 \mathrm{~N}$. For comparison, both systems were used to compute the angular velocity and the angular orientation of the sensor. The VICON system directly computed the angular orientation from the marker positions, while the angular velocity was acquired by differentiation. The gyroscopic data directly represented the angular velocity, while the angular orientation was computed by integrating the angular velocity signal. Due to the lack of a suitable integration constant angular orientations were computed relative to the initial position from the VICON.

Signals were visually compared to assess the quality of the recording. Pearson's linear correlation coefficient was computed between the VICON and Shimmer recordings along all three body axes. Results were averaged for all trials during walking and running, respectively. To test for an eventual asynchronicity between both systems additionally the crosscorrelation function between the signals was computed and used to compare correlation on time-shifted signal versions.

\section{RESULTS}

\section{A. Trigger Accuracy}

Tab. I shows all results from the evaluation of the wireless trigger accuracy. For all transmitter-receiver combinations the mean trigger delay was $2 \mathrm{~ms}$, while the standard deviation was in the range of 20-194 us.

TABLE I

Measured Trigger Delays

\begin{tabular}{|c|c|c|c|c|}
\hline \multirow{2}{*}{$\begin{array}{c}\text { Transmitter } \\
\rightarrow \text { Receiver }\end{array}$} & \multicolumn{4}{|c|}{ Delay (900 trials) } \\
\cline { 2 - 5 } & Mean & Min. & Max. & Std.-Dev. \\
\hline $\mathrm{T} 1 \rightarrow \mathrm{R} 1$ & $2.009 \mathrm{~ms}$ & $1.983 \mathrm{~ms}$ & $2.124 \mathrm{~ms}$ & $18.89 \mathrm{us}$ \\
$\mathrm{T} 1 \rightarrow \mathrm{R} 2$ & $2.009 \mathrm{~ms}$ & $1.986 \mathrm{~ms}$ & $2.126 \mathrm{~ms}$ & $20.19 \mathrm{us}$ \\
$\mathrm{T} 1 \rightarrow \mathrm{R} 3$ & $2.009 \mathrm{~ms}$ & $1.986 \mathrm{~ms}$ & $2.125 \mathrm{~ms}$ & $19.79 \mathrm{us}$ \\
\hline $\mathrm{T} 2 \rightarrow \mathrm{R} 1$ & $2.082 \mathrm{~ms}$ & $1.917 \mathrm{~ms}$ & $2.364 \mathrm{~ms}$ & $53.21 \mathrm{us}$ \\
$\mathrm{T} 2 \rightarrow \mathrm{R} 2$ & $2.082 \mathrm{~ms}$ & $1.895 \mathrm{~ms}$ & $2.368 \mathrm{~ms}$ & $53.09 \mathrm{us}$ \\
$\mathrm{T} 2 \rightarrow \mathrm{R} 3$ & $2.082 \mathrm{~ms}$ & $1.895 \mathrm{~ms}$ & $2.364 \mathrm{~ms}$ & $53.34 \mathrm{us}$ \\
\hline $\mathrm{T} 2 \rightarrow \mathrm{R} 1$ & $2.057 \mathrm{~ms}$ & $1.976 \mathrm{~ms}$ & $3.016 \mathrm{~ms}$ & $193.9 \mathrm{us}$ \\
$\mathrm{T} 2 \rightarrow \mathrm{R} 2$ & $2.020 \mathrm{~ms}$ & $1.978 \mathrm{~ms}$ & $2.157 \mathrm{~ms}$ & $47.65 \mathrm{us}$ \\
$\mathrm{T} 2 \rightarrow \mathrm{R} 3$ & $2.053 \mathrm{~ms}$ & $1.979 \mathrm{~ms}$ & $3.016 \mathrm{~ms}$ & $194.0 \mathrm{us}$ \\
\hline
\end{tabular}

\section{B. Foot Kinematics}

Fig. 3 shows an example recording of the angular velocity and the angular orientation from both systems, together with the corresponding foot-to-ground contact time. Both systems showed a high visual resemblance.
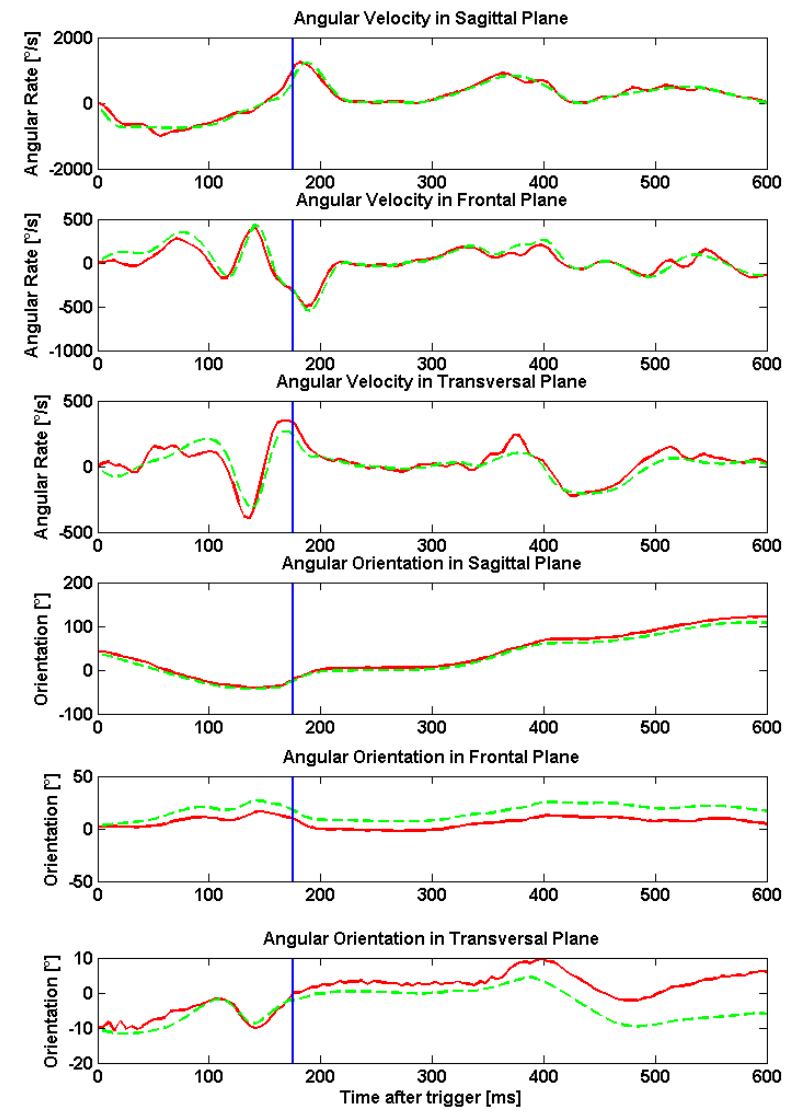

Fig. 3. Example kinematic data acquired during running from the VICON system (red solid lines) and the Shimmer system (green dashed lines) after synchronization. The solid vertical blue line marks the time of foot-toground contact. Both systems showed high correlation.

Tab. II shows the mean correlation in the three axes during the running and walking trials, respectively. Correlation was high in the sagittal plane with a mean correlation coefficient of more than 0.99. The other planes showed a lower correlation with better results in the frontal plane than in the transversal plane. Inspection of the cross-correlation showed that the correlation between the signals was always highest at the original location. Hence no time shift could be found.

TABLE II

CORRELATION TO VICON GOLd STANDARD

\begin{tabular}{|c|c|c|c|c|}
\hline \multirow{2}{*}{ Speed } & \multirow{2}{*}{ Signal } & \multicolumn{3}{|c|}{ Mean Correlation (8 trials) } \\
\cline { 3 - 5 } & & Sagittal & Frontal & Transversal \\
\hline Walking & angular velocity & 0.9773 & 0.7791 & 0.5915 \\
\cline { 2 - 5 } $1.8 \mathrm{~m} / \mathrm{s}$ & angular orientation & 0.9992 & 0.2821 & 0.8001 \\
\hline Running & angular velocity & 0.9731 & 0.8906 & 0.8022 \\
\cline { 2 - 5 } $4.0 \mathrm{~m} / \mathrm{s}$ & angular orientation & 0.9995 & 0.9414 & 0.6388 \\
\hline
\end{tabular}

\section{DISCUSSION}

The evaluation of the trigger accuracy showed that the mean trigger delay of the wireless system was $2 \mathrm{~ms}$, with only slight variations for the sender-receiver combinations. The jitter of the trigger delay over multiple triggers was small for most combinations, resulting in a consistent mean standard deviation of 20 us for sender T1 and 53 us for sender 
$\mathrm{T} 2$. When using sender $\mathrm{T} 1$ or $\mathrm{T} 2$ the mean, minimum and maximum trigger delay was the same for all three receivers, hence all were triggered at the same time. This was not the case for sender T3, which showed inconsistent trigger times and also had a considerably higher standard deviation of 193 us. This shows that the trigger consistency is mainly dependent on the sender module. T1 and T2 showed a small and constant trigger delay with minimum jitter, hence they can be used for the synchronization task. T3 did not meet these requirements. This was probably due to variability in manufacturing or damages during modification, however this must still be evaluated.

The goal of the kinematic evaluation was to demonstrate how the system could be used in a real world synchronous kinematic recording setting. Results showed that the wireless trigger system allowed to synchronize both the inertial sensor data from the sensor node at the heel and the kinematic data from the VICON system. Qualitative inspection of the angular velocity and orientation demonstrated a high visual resemblance between data from both systems. Quantitative evaluation showed a correlation coefficient of more than 0.99 in the sagittal plane. However, the frontal plane during walking and the transversal plane during running had lower correlation. The reason for this was most probably the quite low angular velocity of the foot motion in these planes. This could have led to bad signal to noise ratio in both systems. As the gyroscope range was $2000 \%$ s using a 12-bit ADC, the quantization error was already in the range of $0.5 \%$. This could be solved by using different gyroscopes or sensor fusion. Another problem might be that the segment defined by the markers was not perfectly aligned with the axis of the inertial sensors. This problem could be addressed by a calibration approach. Correlation was higher for the angular orientation than for the angular velocity in some planes, suggesting the gyroscope integration introduced less noise than the VICON differentiation. Contrariwise the orientation in the frontal plane during walking had lower correlation than the angular velocity. This could be related to the used filters or the lower signal to noise ratio in these planes. Data from the accelerometer could not be used, as the maximum range was exceeded during the tests, leading to a saturation of the sensor. This needs to be addressed in future work.

Cross-correlation analysis confirmed that there was no noticeable synchronization error. As the cross-correlation between both systems was always highest at the initial position, we concluded that synchronization was successful within the time resolution of the systems. As the sampling interval of $5 \mathrm{~ms}$ was higher than the average trigger delay of $2 \mathrm{~ms}$, we did not compensate for the trigger delay. However, if the sampling rate was higher, a delay compensation is required. As the trigger delay is nearly constant, this can be achieved by shifting the signals by the mean trigger delay. This is a major advantage, especially when compared to Bluetooth, which has a larger and unstable transmission delay that is hard to compensate.

Compared to other existitng synchronization solutions, the presented wireless trigger system allows highly accurate event synchronization without the overhead of a synchronization protocol. As no constant communication over a wireless channel is needed, this extends battery life and reduces required bandwidth. However, the timing accuracy of the following samples highly depends on the clock and sampling rate stability of the used systems. In our case, the sampling rate of the VICON system was generated by a high accuracy clock in the VICON hardware, but the sampling rate on the Shimmer node was generated by a $32.768 \mathrm{kHz}$ clock crystal, which cannot provide an exact sampling rate of 200 Hz. Hence the synchronicity of both systems will be lost over time. To compensate this a continuous synchronization is required.

In summary the evaluation demonstrated that the system can record foot motion synchronized to external ground truth with high accuracy in a real world setting.

\section{CONCLUSION}

In this paper we have presented a wearable sensor system that allows recording of human foot kinematics with high accuracy ground truth synchronization by combining a shoemounted Shimmer sensor with a wireless trigger device.

We showed that the system can be successfully used for synchronous recording of inertial sensor data and that it had good correlation to external kinematic ground truth. The system had a consistently low trigger delay and very small jitter, making it an ideal choice for external event synchronization. This will allow the creation of a database of human gait data with accurate ground truth synchronization.

Future work will focus on smaller external sensors, the downsizing and integration of the trigger system into Shimmer and a continuous synchronization method for long-term recording.

\section{ACKNOWLEDGMENT}

We wish to thank the supporters of this work, especially Robert Leimer, Roman Janetzko and Simon Sloth. Support was provided by the adidas AG, Herzogenaurach, Germany and the Embedded Systems Institute (ESI) Erlangen.

\section{REFERENCES}

[1] M. Chen et al., Body Area Networks: a Survey, Mobile Netw. Appl., vol. 16, 2, April 2011, pp. 171-193.

[2] A. Burns et al., SHIMMER - A Wireless Sensor Platform for Noninvasive Biomedical Research, IEEE Sensors. vol. 10, no. 9, Sept. 2010, pp. 1527-1534.

[3] E. Tapia at al., Activity Recognition in the Home Using Simple and Ubiquitous Sensors, Perv. Comp., vol. 3001, 2004, pp. 158-175.

[4] A. Salarian et al., Gait assessment in Parkinson's disease: toward an ambulatory system for long-term monitoring, IEEE Transactions on Biomedical Engineering, 2004, pp. 1434-1443.

[5] S. Patel et al., Monitoring motor fluctuations in patients with Parkinson's disease using wearable sensors, IEEE Transactions on Information Technology in Biomedicine, vol. 13, 2009, pp. 864-873.

[6] B. Mariani et al., 3D gait assessment in young and elderly subjects using foot-worn inertial sensors. Journal of Biomechanics, vol. 43, 2010, pp. 2999-3006.

[7] B. K. Higginson, Methods of running gait analysis, Curr. Sports Med. Rep., vol. 8, no. 3, 2009, pp. 136-141.

[8] B. M. Eskofier et al., Classification of perceived running fatigue in digital sports, Proc. Intl. Conf. on Pattern Recognition (ICPR 2008), Dec. 2008, pp. 1-4.

[9] J. Barth, et al. Biometric and Mobile Gait Analysis for Early Diagnosis and Therapy Monitoring in Parkinson's Disease, Intl. Conf. IEEE Engineering in Medicine and Biology Society (EMBC 2011), 2011, pp. $868-871$.

[10] J. Klucken et al., Mobile biosensor-based gait analysis: A diagnostic and therapeutic tool in Parkinson's disease, Der Nervenarzt, 2011.

[11] J. Rueterbories et al., Methods for gait event detection and analysis in ambulatory systems, Med. Eng. Phys., vol. 32, no. 6, 2010, pp. $545-552$.

[12] K. Holger et al., Protocols and Architectures for Wireless Sensor Networks, John Wiley \& Sons, 2005. 